\title{
La constitución de las políticas de investigación e innovación responsables: tensiones en la instrumentalización y la regulación
}

\author{
Andoni EizagirRe \\ Mondragon Unibersitatea \\ aeizagirre@mondragon.edu
}

Recibido: 01-04-2015

Aceptado: 25-11-2015

\begin{abstract}
Resumen
La política científica y tecnológica europea ha propuesto alinear mejor el proceso y los resultados de las actividades de investigación e ingeniería con los valores, las necesidades y las expectativas de la sociedad europea. No obstante, esta progresiva radicalización de la narrativa, como respuesta a las demandas de mayor responsabilidad, debe lidiar con otros discursos del presente que operan como prescripciones ideológicas y conciben la innovación como motor de la competitividad económica. Hay una tensión de base que se evidencia en la aparente paradoja del nuevo contexto social de la actividad científicotecnológica sometida, por un lado, a una creciente demanda de modelos responsables de investigación e innovación y, por otro, instrumentalizada para resultados macroeconómicos. Nuestra estrategia de análisis sugiere rescatar esta cuestión ligada a los intereses que concurren en la generación del conocimiento, los criterios que rigen las dinámicas del régimen científico-tecnológico y los valores que orientan las políticas científicas. En la tensión de base encontramos una infinidad de elementos heterogéneos que en ocasiones se contradicen y que disputan entre sí por formas alternativas de instrumentalizar y regular las dinámicas del sistema de ciencia, tecnología e innovación. En la gobernanza de esa tensión se constituye el modelo de investigación e innovación que las políticas europeas y nacionales desarrollan como respuesta a los desafíos globales.
\end{abstract}

Palabras clave: Investigación e innovación responsables; política; valores; gobernanza.

\section{The Constitution of a Responsible Research and Innovation Policy: Tensions in Implementation and Regulation}

\begin{abstract}
European scientific and technological policy recommends better aligning the process and results of activities of research and engineering with the values, needs, and expectations of European society. Nevertheless, this progressive intensification of the narrative, as a response to demands for greater responsibility, must contend with other present discourses that function as ideological prescriptions and envisage innovation as a motor for economic competitiveness. There is a basic tension that is clear in the apparent paradox of the new social context of scientific-technological activity, which, on the one hand, is subject to a growing demand for responsible models of research and innovation, and on the other hand, is implemented for macroeconomic results. Our analysis strategy is to reclaim this issue, which is closely linked to the interests that intersect in the generation of knowledge, the criteria that govern the dynamics of the scientific-technological system, and the values that guide scientific policy. Within this basic tension we find a myriad of heterogeneous elements that sometimes contradict each other and that compete amongst themselves for alternative ways to implement and regulate the dynamics of the system of science,
\end{abstract}


technology and innovation. The model of research and innovation developed by European and national policies in response to global challenges is created through the management of this tension.

Key words: responsible research and innovation; policy; values; governance.

\section{Referencia normalizada}

Eizagirre, A. (2016): "La constitución de las políticas de investigación e innovación responsables: tensiones en la instrumentalización y la regulación”, Política y Sociedad, 53 (3), pp. 815-836.

Sumario: Introducción. 1. Las políticas de estímulo de la ciencia. 2. Las políticas de regulación de la ciencia. 3. La promesa del beneficio en cuestión. 4. La promesa de la seguridad en cuestión. 5. Consideraciones finales. 6. Bibliografía.

\section{Agradecimientos}

La elaboración de este artículo ha contado con el apoyo del Departamento de Educación, Política Lingüística y Cultura del Gobierno Vasco (IT644-13).

\section{Introducción}

En los últimos años el contexto en el que operan la actividad científica y tecnológica ha adquirido rasgos distintivos. Entre las particularidades destacan la relevancia que poseen los criterios prácticos y las expectativas instrumentales en la organización, la financiación y la valoración del sistema de ciencia, tecnología e innovación. A este hecho se une la generación de normas que como respuesta a la conciencia de los riesgos y de los efectos indeseados regulan la producción, la distribución y la comercialización del conocimiento. También se observan cambios en las interacciones con una sociedad crecientemente exigente y crítica ante las dinámicas que estabilizan el régimen científico-tecnológico.

El punto de partida del artículo radica en esa aparente paradoja del nuevo contexto social de la actividad científico-tecnológica sometida, por un lado, a una creciente demanda de modelos responsables de investigación e innovación $y$, por otro, instrumentalizada para resultados macroeconómicos. Para su elucidación se propone una reconstrucción crítica de las PCTI y su relación con la sociedad. La hipótesis general se formula como sigue: las recientes iniciativas conceptuales, metodológicas y organizacionales sobre las PCTI están supeditadas a su dimensión estructural e institucional y orientadas a la optimización del conocimiento generado, por lo que se subestima cualquier discusión centrada en los motivos que originan la producción del conocimiento, su aplicación y su gestión y se minimizan los aspectos ligados a la necesidad, la deseabilidad y la aceptabilidad social. Nuestra estrategia de análisis sugiere rescatar la cuestión ligada a los intereses que concurren en la generación del conocimiento, los criterios que rigen las dinámicas del régimen científico-tecnológico y los valores que orientan las PCTI. Es irónico que a la vez que se reconoce la naturaleza compleja de la innovación las propiedades normativas de la trayectoria socio-tecnológica en áreas como la energía, el transporte, la agricultura, la salud y la 
alimentación se decidan sobre un conjunto limitado de intereses e intenciones sociales. Para expresarlo en términos de la teoría evolucionista de la innovación, veremos que las preferencias en el proceso multidireccional de variación y selección que consolidan las formas científico-tecnológicas se rigen mayormente por la utilidad y la rentabilidad de mercado.

En este artículo vamos a vincular con un enfoque crítico las dimensiones de la promoción y de la regulación de las PCTI. Por ello, el artículo contiene dos principales bloques, uno relativo a la evolución de las PCTI, y otro que suministra elementos críticos y contribuye al debate. En ambos casos, los bloques se estructuran alrededor de las dos dimensiones de las PCTI: por una parte, las políticas orientadas al estímulo del conocimiento y el cambio tecnológico; por otra parte, las bases legales e instrumentos analíticos para prever los impactos de las tecnologías e intentar mitigarlos. Esta distinción analítica nos permite comprender mejor dos de las razones que dificultan transformar las relaciones políticas entre la actividad científico-tecnológica y la sociedad, una ligada al ámbito de la instrumentalización, y otra al de la regulación: por una parte, el contexto social de la investigación y la innovación, que está manifiestamente reducido por la rentabilidad de mercado; por otra parte, una concepción ingenua de la ciencia, que presupone una visión científica sobre el progreso así como la traslación automática de la verdad y la certidumbre (distinguidos como el código de la ciencia) a la racionalidad política.

Este artículo defiende la siguiente tesis: un modelo de gobierno responsable debe interesarse por los objetivos a perseguir, los motivos que originan particulares formas de organizar, generar y validar la investigación y la innovación, así como los mecanismos que facilitan la apertura al pluralismo y las propuestas alternativas.

\section{Las políticas de estímulo de la ciencia}

La política científica en su origen asume una visión científica del progreso y un modelo basado en el empuje de la ciencia. En el informe Science: The Endless Frontier dirigido por Bush (1945) podemos encontrar los supuestos y contenidos que asume el primer modelo canónico de las políticas científicas: 1) el conocimiento generado a través de la investigación básica está en el origen de la innovación y la difusión; 2) se fija una financiación sin límites de la investigación, no condicionada por objetivos, y proyectada a largo plazo; 3 ) se establece una asociación secuencial y triunfalista entre investigación básica, aplicación tecnológica y beneficio ilimitado en los ámbitos de la seguridad, el ambiente y el bienestar; y 4) se garantizan la incentivación de la carrera investigadora, la autonomía de los científicos en su objetivo de aumentar el acervo de conocimiento existente, y su independencia (funcional y moral) ante las aplicaciones tecnológicas del conocimiento.

En las próximas décadas esta política de patronazgo es ampliamente discutida, se cuestionan los supuestos de origen ligados a ese modelo lineal, las indagaciones empíricas detectan ámbitos de investigación que responden con mayor flexibilidad a las exigencias de mercado, a la vez que se asume la necesidad de adecuar las prioridades entre la oferta y la demanda. Este mayor interés por los resultados de mercado prima 
aquellos mecanismos de aprovechamiento de la inversión y líneas estratégicas que aseguren la transferencia de conocimiento al ámbito del sistema productivo. ${ }^{1}$ De esta manera, bajo criterios de optimización de recursos y una ciencia sensible a su dimensión aplicativa y estratégica, se inician los primeros cambios en los sistemas de investigación y desarrollo. Con todo, todavía a principios de la década de 1970, un comité de la OCDE coordinado por el ingeniero Harvey Brooks en su informe Science, Growth and Society: A New Perspective (OECD, 1972) formulaba un "sistema de ciencia, tecnología y sociedad" claramente orientado a satisfacer las demandas sociales $\mathrm{y}$ al bienestar de la sociedad. Conviene retener ambas puntualizaciones.

De hecho, la nueva orientación de las PCTI se alimenta de los problemas empíricos del modelo lineal, que obliga a realizar cambios en el sistema de ciencia y en su organización, pero al mismo tiempo impone unos objetivos crecientemente orientados por la demanda de mercado. Como bien advierte Kallerud (2012), una cosa es la necesidad de reformular el modelo lineal tal y como lo sugirió ingenuamente el informe de Bush, y cosa bien distinta es aprovechar la optimización de recursos y los cambios en el sistema de investigación para alterar los objetivos y redefinir los resultados. La distinción de ambas dimensiones revela que el reordenamiento bajo criterios técnicos ligados a la relación entre ciencia, tecnología y sociedad ha enmascarado un giro en las PCTI. La hegemonía de los estudios de innovación ha oscurecido esta distinción analítica y se ha omitido la tensión sobre la dirección y el contenido de la investigación y la innovación. Si nos referimos nuevamente al informe elaborado por Brooks, también podemos comprender que el avance de la ciencia y sus motivos paulatinamente se desvinculan de sus efectos en la expansión de las capacidades de las personas, como respuesta de las necesidades humanas, y como solución de problemas como la energía, el medio ambiente y la ciudad (Miettinen, 2013).

Buena parte de estas discusiones sobre el diseño y la implementación de las PCTI se enmarcan en los problemas que la innovación tecnológica impone en la década de 1970, un momento histórico que además se caracteriza por otros cambios más amplios ligados a las formas de regulación de las economías capitalistas (Barben, 2007; Jamison, 2012). Salomon (2000) y Godin (2007) han confirmado que la retórica institucional sobre la ciencia y su desarrollo en los distintos sistemas nacionales y regionales está subordinada al lenguaje político que fabrican la National Science Foundation (NSF) y sobre todo la Organización para la Cooperación y el Desarrollo Económico (OCDE) a través de los procedimientos para la selección y recogida de datos estadísticos de ciencia y tecnología y los indicadores para su medición.

Un análisis riguroso de los informes y documentos de la OCDE resuelve que el eje de comprensión se desplaza de reconocer la dimensión económica de la ciencia a considerar en los datos estadísticos, en los indicadores sobre productividad científica y

${ }^{1}$ Entre los documentos que impulsan este cambio del modelo de empuje de la ciencia al modelo de demanda de mercado: OCDE. 1968. Gaps in Technology: General Report. Paris: OCDE; OCDE. 1971. The Conditions of Success in Technological Innovation. Paris: OCDE; OCDE. 1972. The Research System. Paris: OCDE. Para un análisis del modelo de demanda mercado: Godin y Lane, 2013. 
resultados tecnológicos, en los criterios de valoración de los proyectos, así como en las medidas políticas y legales aspectos ligados principalmente a la economía y el impacto económico. ${ }^{2}$ En la misma línea, la importancia para la economía de las relaciones entre ciencia y tecnología se refleja en el interés por los modelos econométricos y su uso en las discusiones relativas a la política científico-tecnológica y su agenda (Godin, 2005). A todo ello se unen la consolidación de nuevas teorías económicas sobre el crecimiento y de enfoques funcionalistas y sistémicos de la innovación. Como resultado, sobresalen los cambios en los valores e intereses concurrentes que rigen la investigación y el desarrollo (dominio de la cultura empresarial en la organización y práctica de la ciencia, gasto público orientado a la empresa); en el sistema y sus estructuras (consolidación del "sistema de ciencia, tecnología y empresa", bajo el acrónimo "i+d+i"); y en los estilos de gestión política (ministerios de economía y secretarías de Estado sobre innovación que asumen competencias relativas a la ciencia y la educación superior).

En este contexto, los análisis críticos ligados a la actividad científico-tecnológica y su organización ponen énfasis en la consolidación del modelo neoliberal de regular la economía capitalista (Moore et al., 2011; Slaughter y Rhoads, 2004; Stephan, 2012). A este respecto Berman $(2012,2014)$ hace una importante precisión: Berman observa que la dependencia por la nueva retórica se encuentra en gobiernos de tradición diversa y concluye que el factor explicativo es más bien la agudización generalizada de la lógica economicista. Esta puntualización creemos que sirve para comprender la mutación en las PCTI como resultado de una interacción de fenómenos: a) la economía adquiere primacía en la política y hay un replanteamiento generalizado de las políticas públicas en términos de su impacto económico; b) se apuesta por definir la ciencia y la tecnología como recurso económico y por extensión los criterios que valoran y organizan la

${ }^{2}$ Los informes de la ODCE agrupados bajo "Tecnología y política económica" sitúan explícitamente las teorías sobre el cambio tecnológico en el núcleo de la política económica. Se trata de los siguientes documentos: OCDE. 1991. Choosing Priorities in Science and Technology. Paris: OCDE; OCDE. 1991. Technology in a Changing World. Paris: OCDE. OCDE. 1992. Technology and the Economy: the Key Relationships. Paris: OCDE. En los 90s se elaboran los primeros indicadores que orientan el cambio y se centran en las transacciones comerciales y los datos de patentes, e incluso despuntan los indicadores no bibliométricos para el análisis de la productividad científica. El lenguaje político contenido en la sociedad del conocimiento (OCDE. 1996. The Knowledge-Based Economy. Paris: OCDE) justifica la retórica ligada a la extensión los cambios estructurales de la economía, la liberalización creciente de los intercambios comerciales, la deslocalización de actividades productivas (principalmente en el sector industrial) y las nuevas estructuras políticas de regulación comercial. Por último, en los siguientes años la nueva literatura sobre innovación consolida definitivamente este horizonte ideológico. Véanse los siguientes documentos: OCDE. 1999. Managing Innovation Systems. Paris: OCDE; OCDE. 2001. Innovative Networks: Co-operation in National Innovation Systems. Paris: OCDE; OCDE. 2001. Innovative Clusters: Drivers of National Innovation Systems. Paris: OCDE; OCDE. 2001. Innovative People: Mobility of Skilled Personnel in National Innovation Systems. Paris: OCDE; OCDE. 2002. Dynamising National Innovation Systems. Paris: OCDE; OCDE. 2004. Science and Innovation Policy: Key Challenges and Opportunities. Paris: OCDE; OCDE. 2005. Governance of Innovation Systems. Paris: OCDE. 
actividad científica y tecnológica son también de naturaleza principalmente económica; c) el lenguaje político transforma la ciencia en una dimensión clave de la economía y de la actividad económica; d) se consolida una comunidad científica que interactúa con los economistas de la innovación y asume que las PCTI determinan la productividad y la competitividad (Godin, 2015: 261-280). A todo esto se une que entre los discursos del presente que operan como prescripciones ideológicas, sobresalen la innovación y la sociedad del conocimiento, que como relato cultural enmarcan las tecnologías emergentes como el nuevo motor -como fueron la energía de vapor y el motor eléctricode los cambios sociales (Reynolds y Szerszynski, 2012).

Todo ello ha tenido una serie de consecuencias: por una parte, se consolida una concepción reduccionista, determinista y solucionista de la innovación, que naturaliza la tecnología y estabiliza un régimen tecno-económico; por otra parte, las medidas dirigidas a alimentar el flujo de resultados entre el sistema de producción de conocimiento y el sistema de producción de bienes y servicios orientan claramente la balanza a consideraciones de política económica (Marklund, Vonortas y Wessner, 2009). ${ }^{3}$ De esta manera también se supedita la discusión sobre los objetivos, que se ha orientado por las necesidades impuestas por el mercado. Finalmente, las PCTI y su gobernanza han venido desarrollando un rol importante en la legitimación de la racionalidad económica.

No obstante, esta crítica es asumida, aunque de manera parcial, en las recientes iniciativas sobre investigación e innovación. En este sentido, un análisis de los principales documentos de las distintas organizaciones internacionales sugiere que la noción de desafíos globales se ha convertido en uso común en los objetivos y motivos políticos que se enmarcan para apoyar, financiar y movilizar la investigación y la innovación. De esta manera, se asume la necesidad de ampliar el debate, hasta ahora contenido en las dimensiones institucionales (organización y funcionamiento de los sistemas de PCTI, sus instituciones e instrumentos, la optimización de recursos, la gestión del conocimiento generado), también a los objetivos y valores de la PCTI. ${ }^{4}$

\section{Las políticas de regulación de la ciencia}

Junto a las políticas de estímulo, a partir de 1970 la otra piedra angular de las PCTI es la regulación del conocimiento y de la aplicación tecnológica, en tanto que establece

${ }^{3}$ Dos buenos análisis complementarios son Elzinga, 2012 y Hackett, 1990.

${ }^{4}$ Este cambio se observa en la Unión Europea, en el giro discursivo que supone el "Horizonte 2020" y la iniciativa "Unión por la Innovación", pero también en las políticas de innovación de la OCDE y en la estrategia de Obama. Véanse: EC. 2011. Horizon 2020 - The Framework Programme for Research and Innovation. 30.11.2011. COM(2011) 808 final, Brussels; EC. 2013. Horizon 2020, Work Programme 2014-2015: 16. Science with and for Society, C(2013) 8631, Brussels; OECD. 2010. The OECD Innovation Strategy: Getting a Head Start on Tomorrow. Paris: OECD; Obama, B. 2009. A Strategy for American Innovation. National Economic Council and Office of Science and Technology Policy. (Este último documento se ha actualizado en 2011 y en 2015) 
mecanismos expertos y legislativos de control, determina y garantiza niveles aceptables de riesgo para la sociedad, y por último legitima socialmente el progreso tecnológico e industrial. El análisis de las políticas de regulación nos permite completar la imagen como se ha conceptualizado la innovación científico-tecnológica y su relación con el progreso social.

Un análisis exhaustivo y riguroso de las distintas modalidades de regulación de los desarrollos científico-tecnológicos e industriales indica que la reacción de las administraciones tradicionalmente se ha basado sobre la determinación de impactos y el cálculo de riesgos (Shrader-Frechette, 2007). La respuesta a los impactos adversos ha institucionalizado la regulación basada en el análisis del riesgo, que posterga su estudio a modelos retrospectivos con base en las evidencias que científicos y expertos disponen.

El modelo estándar de análisis del riesgo incorpora los ámbitos de evaluación y de gestión. La fase de la evaluación asume una concepción de riesgo calculable y se compromete a establecer niveles de tolerabilidad del riesgo. Primero se identifica aquello que supone una amenaza para la salud y la seguridad humana, luego se estiman la probabilidad y la magnitud de los daños asociados, y finalmente se evalúa el nivel de aceptabilidad del riesgo. La actividad evaluadora en su definición de quehacer científico, es una labor de asesoramiento sobre riesgos (Shrader-Frechette, 1991: 5556), y se orienta por la caracterización del problema, la evaluación de su efecto, la recopilación de los valores afectados, la identificación de los mecanismos implicados y la formulación de métodos para la aplicación de factores de seguridad. A la evaluación le sigue una fase de gestión que asume una concepción de riesgo imputable y se compromete a establecer las necesarias garantías jurídicas.

Esta segunda tarea consiste en evaluar y ponderar las alternativas existentes a la luz de los resultados obtenidos en la fase de la evaluación. En este sentido, los organismos reguladores y los legisladores también deben certificar que la exposición al riesgo cumple los estándares de tolerabilidad determinados en la fase de evaluación, y para ello, toman las medidas políticas y legales oportunas como, por ejemplo, las inspecciones y las sanciones (McDaniels y Small, 2004). Una vez que los riesgos son anticipados y delimitados localmente, temporalmente y socialmente, deben ser imputables conforme a normas de causalidad, responsabilidad y culpa. A ello se une una concepción del riesgo compensable y asegurable. Esta lógica de la indemnización asume un sistema de la seguridad mediante compensación anticipada. La dimensión normativa del riesgo se explica ciertamente por esta controlabilidad que los mecanismos expertos y legislativos anticipan y gestionan.

A partir de la década de 1980 se consolida la fase denominada comunicación del riesgo, que en base a un modelo típicamente unidireccional y divulgativo, tiene como objetivo informar de los hechos y confiar de la actuación de las instituciones, en clara respuesta a -según el diagnóstico institucional- la creciente aversión al riesgo y a las percepciones subjetivas disfuncionales y originarias de controversias sociales paralizantes (Otway y Wynne, 1989).

La esencia del riesgo, como bien sintetiza Renn (1992), comprende un carácter descriptivo y normativo, es decir, indica la posibilidad de efectos indeseados causados 
por una acción, incorpora la idea de una relación causal entre la actividad humana y/o tecnológica y las consecuencias posibles que pueda inducir, en tanto que su objetivo es transformar las causas y atenuar las consecuencias no deseadas, y por tanto, el riesgo interioriza la determinación de los impactos que puede causar una acción. En el análisis del riesgo -presupone la visión tecnocrática- la ciencia y los expertos, a través de las leyes de racionalidad y reglas normativas, garantizan el conocimiento verdadero, y su capacidad de control y predicción representa la certidumbre como garantía de la actividad científica y del proceso regulador.

Las implicaciones sociales y políticas de esa concepción del riesgo (calculable, imputable y compensable) son contundentes. En primer lugar, el futuro sociotécnico queda bajo la asignación de probabilidades numéricas, en tanto que aquello definido como situación de riesgo resulta cuantificable, siendo incluso la información que se dispone imperfecta. En efecto, la adopción del análisis técnico posibilita, justamente, definir un suceso futuro como riesgo y por tanto evaluar la probabilidad de las consecuencias de las acciones tecnológicas y su magnitud. El riesgo impone esta capacidad de asignar probabilidades y la severidad del daño. En segundo lugar, su caracterización institucional identifica el riesgo como un hecho excepcional y aislado; la baja probabilidad de que surjan accidentes da lugar a la expresión "riesgo cero en la práctica". En tercer lugar, la concepción numérica del riesgo establece una distinción entre el riesgo objetivo y su percepción social, a la vez que desvincula cualquier aspecto social, moral y ambiental no traducible a una dimensión técnica, objetiva y medible. Es más, el análisis del riesgo rompe con la tradición determinista que presumía el modelo de impacto ambiental y adquiere preferencia una concepción más bien probabilística. Además, es importante señalar que es en este marco normativo en el que se fijan también los códigos de conducta responsables en la actividad de científicos e ingenieros, las reglas y normas ligadas a su integridad, al mismo tiempo que cierra técnicamente los escenarios de seguridad tecnológica e industrial.

Por último, esta caracterización de las formas de gobernar la ciencia subordina la sociedad a las políticas diseñadas e implementadas. El sentido moral que se asigna a la gente de negocios y a los científicos, que traduce la responsabilidad como un acto racional dependiente de los resultados (ética consecuencialista), confiere un lugar secundario a todo lo demás. Así, una concepción normativa de la ciencia que asocia automáticamente avance del conocimiento y progreso social omite el escrutinio público de las PCTI. De hecho, como puntualizan entre otros Kallerud y Ramberg (2002), los estudios sociológicos sobre la percepción social de la ciencia han estado sujetos al modelo de déficit cognitivo, según el cual hay una correlación entre nivel de conocimiento del vocabulario y método científico y las percepciones sociales.

Así, se asume una definición normativa de ciudadano científicamente informado y somete toda política de comunicación social a modelos divulgativos orientados a promover la alfabetización cívica de la ciencia. ${ }^{5}$ Esta explicación se maneja sobre todo

${ }^{5}$ Para una valoración de los estudios sociológicos sobre percepción social de la ciencia: Bauer 2012, 2015; Besley, 2013; Eizagirre, 2013; Laspra y Muñoz, 2014 von Roten y de Roten, 2013. 
cuando emergen conflictos socio-ambientales y la élite político-económica censura la oposición argumentando que en su seno brotan -para expresarlo con Douglas- "el desconocimiento" y "el ventajismo ideológico", de tal manera que los riesgos socioambientales se reducen a mero producto de la subjetividad ideológica-cultural. ${ }^{6}$

\section{La promesa del beneficio en cuestión}

La segunda parte del artículo expone que las PCTI no pueden entenderse como un proceso lineal en el que ideas brillantes se traducen en resultados predecibles y sujetos a gestión. Por el contrario, encontramos una infinidad de elementos heterogéneos que en ocasiones se contradicen y que disputan entre sí por formas alternativas de instrumentalizar y regular las dinámicas del sistema científico y técnico (Todt, 2011). A esta pluralidad de intereses debemos agregar la paulatina consolidación de una sociedad crecientemente exigente y crítica ante las dinámicas que estabilizan el régimen científicotecnológico. En los siguientes dos apartados discutimos las premisas que incorporan las políticas de estímulo y regulación, y sus respectivas promesas de beneficio ilimitado y seguridad. En este apartado afrontamos los problemas de las políticas de estímulo y de las promesas de beneficio ilimitado.

Hay una ambigüedad no resuelta en la retórica ligada a las PCTI. Un análisis detenido nos permite identificar simultáneamente la institucionalización del cambio y de la innovación, el intervencionismo económico, la reproducción sistemática de las promesas de crecimiento y bienestar, y el discurso de la no-interferencia por el carácter impredecible de la ciencia y su desarrollo. Así, a la vez que el conocimiento y la innovación se asocian a promesas de futuro y por extensión se justifica su financiación, cualquier sugerencia relativa a los mecanismos de gobierno tropieza con la retórica sobre la autonomía del científico. ${ }^{7}$ Se trata de un aspecto clave omitido en los estudios sociales de la ciencia y que de manera acrítica también integra la gobernanza de las PCTI en sus diferentes variantes aplicativas. Conviene aclarar las contradicciones que en su seno integra un discurso de este tipo.

La discusión remite necesariamente a Bernal (2010), que en oposición a la tesis de Polanyi (1962), sostuvo en 1939 una concepción de la ciencia instrumental para la transformación social y la emancipación, una ciencia enraizada en la vida práctica. Esta perspectiva indica que la ciencia debe ser valorada por su utilidad al bienestar de la sociedad, una libertad científica sujeta a políticas de investigación orientadas por el

\footnotetext{
${ }^{6}$ Hay en los estudios sociales del riesgo una tradición que comprende el riesgo como mero artefacto cultural, en su caso instrumentalizado por los grupos ecologistas para sus intereses ideológicos. Esta tradición comprende que la dimensión política del riesgo está ligada exclusivamente al interés de un grupo de consolidar su posición en la sociedad (Douglas y Wildavsky, 1982).

${ }^{7}$ Es frecuente recurrir a la tesis de Polanyi (1962: 62): "Se puede matar o mutilar el avance de la ciencia, pero no modelarla. Ella sólo puede avanzar por pasos que son esencialmente impredecibles, buscando problemas propios, y los beneficios prácticos de estos avances serán siempre incidentales $\mathrm{y}$, por lo tanto, doblemente imprevisibles".
} 
bienestar objetivo de las personas, a la vez que la curiosidad intelectual y desinterés social del científico debe ser reemplazado por una obligación ética ante conocimientos beneficiosos para la sociedad. ${ }^{8}$ Por el contrario, el acervo cultural que despuntó en la segunda mitad del siglo XX ancló en el imaginario social de la ciencia una imagen bien diferente.

Una extraña alianza implícita entre diferentes retóricas, como sugieren Elzinga y Jamison (1995), resolvió la disputa: la retórica promovida por los científicos naturales sobre la investigación básica, los industriales sobre el desarrollo tecnológico y los economistas sobre la innovación racionaliza bajo instrumentos tecnocráticos el paradigma lineal y triunfalista. Se trata de un discurso que además casa bien con la lógica economicista y el régimen tecnocientífico emergente. De hecho, los tres ejes bajo los que se ha articulado el discurso liberal (la ciencia, la democracia y el mercado) avanzan el resultado siguiente: un discurso que liga simultáneamente la impredecibilidad de los descubrimientos científicos y la justificación de las políticas de investigación sobre la base de los beneficios anticipados; una serie de promesas iniciales que a falta de una evaluación de los resultados alimenta la imagen triunfalista de la ciencia; una abierta competición entre científicos por la obtención de recursos generalmente condicionados a líneas de investigación y aplicación previamente orientados; una reducción del beneficio social al beneficio económico. Como resultado final, se mantiene intacto el principal dogma de las PCTI, a saber, el beneficio social de la ciencia es inherente a la ciencia misma, al mismo tiempo que la asignación y organización de los recursos intelectuales y fiscales que posibilitan la investigación adquieren un carácter estratégico y se rigen por criterios prácticos.

Todo indica que, como advirtió de manera prematura Dickson (1984), la impredecibilidad de los resultados termina siendo un arma arrojadiza frente a quienes proponen una discusión sobre los objetivos a perseguir y la necesidad de cuidar los efectos adversos e imprevistos de la actividad científico-tecnológica, bajo el postulado de que ambas exigencias se desvinculan de los beneficios inherentes a la generación del conocimiento e incorporan factores extrínsecos a la ciencia. Esto explica finalmente dos aspectos reveladores: por una parte, la autonomía y la efectividad de la empresa científica opera sobre mecanismos de control interno (revisión por pares) y se mide sobre criterios internos (inversión y financiación, publicaciones, investigadores, patentes, reconocimientos y premios); por otra parte, sin embargo, la retórica sostenida transforma estos resultados internos en beneficios exteriores a la sociedad, de tal manera que la métrica incluye los outputs (medición de la actividad), pero no así los resultados (outcomes). ${ }^{9}$ Aunque parezca extraño, como Sarewitz viene reiterando sucesivamente

\footnotetext{
${ }^{8}$ Algunas décadas más tarde Ziman (1998: 1813-1814) ha concluido que "la ética no es sólo una disciplina intelectual abstracta. Trata de los conflictos que surgen al intentar satisfacer las necesidades humanas y los valores reales. El ethos de la ciencia académica oficial sistemáticamente excluye todas esas consideraciones".

${ }^{9}$ Hay un fenómeno adicional. Godin ha reflexionado sobre todas estas cuestiones a través de un análisis crítico de los indicadores y ha comprobado que los indicadores sobre impacto no-económico son escasos. Las pocas excepciones son estudios sobre el impacto de la ciencia
} 
(Bozeman y Sarewitz, 2011; Sarewitz, 2007; Sarewitz et al., 2004; Woodhouse y Sarewitz 2007), bajo una concepción descontextualizada de la ciencia, la evaluación de las promesas y los resultados no ha merecido en líneas generales particular interés, lo que libra la retórica de los beneficios ilimitados a someterse a una evaluación de los resultados.

Aquí podemos anticipar una primera crítica general de las PCTI. Si bien a lo largo de las últimas décadas han cambiado las formas de producir, organizar y distribuir el conocimiento, hay un imaginario que se articula en discursos positivistas y promesas triunfalistas, pero que carece de fundamento empírico, o cuando menos la ciencia de la política científica es marginal y no tiene reflejo en las decisiones políticas (Coozens et al., 2007). En segundo lugar, esto ha permitido descuidar el hecho de que la retórica triunfalista y positivista del avance científico-tecnológico enmascara el factor economicista en la redirección de lo que debe ser promocionado y estimulado, a la vez que omite cualquier crítica de la comercialización del conocimiento y del desinterés por los efectos adversos. Se alimentan mutuamente las promesas, las inversiones y una reorganización general de las tecnologías emergentes y convergentes, al tiempo que sus efectos de carácter multidimensional son excluidos (Felt, 2007)..$^{10}$ En definitiva, hay una comprensión de la ciencia exclusivamente en términos de beneficio, entendido en términos macroeconómicos, lo que advierte un tipo de reduccionismo científico donde la economía -y una economía de cierto tipo- es la que al final tiene los criterios de valoración de la empresa científica.

Todo esto guarda poca relación con las paradojas que encierran en su seno las PCTI: se omite la discusión y evaluación de sus efectos adversos e imprevistos; se enmascaran los motivos y objetivos que guían la ciencia financiada e instrumentalizada; se desestiman sin discusión aquellas disciplinas y ámbitos de investigación que no inducen atracción; se minusvalora la relación entre resultados económicos y sus implicaciones en otras esferas de la vida social. ${ }^{11}$ Es llamativo que la comprensión y

sobre sí misma (el impacto de las publicaciones científicas en otros investigadores) y sobre la innovación tecnológica (el impacto de la investigación académica sobre el avance y desarrollo de la innovación industrial). Algunos investigadores, conscientes de las limitaciones señaladas, han tratado de ampliar el análisis de los impactos hacia aspectos más indirectos (incremento del acervo de conocimientos útiles, capacitación de graduados universitarios, creación de nuevos instrumentos y metodologías, formación de redes y estímulo de interacciones sociales). Se trata de algo intermedio, pero un análisis integral sobre el valor que tiene el sistema de investigación en la sociedad debe necesariamente medir también el impacto de la ciencia sobre otras dimensiones de la realidad socio-natural (Godin, 2010; Godin y Doré, 2005).

${ }^{10}$ De hecho, cabe remarcar el rol que juegan las promesas y las expectativas en la formación y estímulo del cambio científico y tecnológico, en tanto que las abstracciones orientadas al futuro guían actividades, proporcionan estructuras y legitimación, atraen interés y fomentan inversión (Brown, 2003; Brown y Michael, 2003; Brown et al., 2006; Coenen y Simakova, 2013; Horst, 2007; Selin, 2007).

${ }^{11}$ Cuestiones de este tipo se identificaron en la "World Conference on Science for the Twenty-First Century: a New Commitment", celebrado en Budapest entre el 26 de junio y 
la evaluación de las interacciones entre las decisiones sobre las PCTI y sus resultados (no económicos) hayan merecido tan escaso interés, en buena parte descartado por las diferentes autoridades bajo el dogma de su imposibilidad, pero que no obstante interfiere en todos los ámbitos institucionales y sociales. ${ }^{12}$

En este orden de cosas, hay otros elementos adicionales que debemos rescatar para completar el debate. En primer lugar, los estudios sociales del conocimiento han demostrado concluyentemente la relevancia que los condicionantes sociales -y no sólo los epistémicos- tienen en la generación, distribución y regulación del conocimiento. En segundo lugar, la contribución que acomete la ciencia se enmarca en un contexto político, cultural y económico, en el que concurren de manera asimétrica intereses diversos y dispares. En tercer lugar, la ciencia nunca se desarrolla en el vacío social y político, de tal manera que las promesas obligan a cambios diversos en ámbitos como la educación y en los sectores productivo, financiero y crediticio, condición necesaria para el desarrollo óptimo de la actividad investigadora (es importante señalar que de esta manera también se re-introduce el modelo lineal de innovación). En cuarto lugar, el conocimiento científico no es mera comprensión del mundo, sino que su comprensión nos permite también intervenir en el mundo y de esta manera alterarlo. En quinto lugar, las PCTI se han diseñado e implementado en economías basadas en el crecimiento, el petróleo abundante y barato, y la deuda.

Todas estas líneas críticas redundan en el principal resultado que hemos anticipado: una cosa es remarcar la creciente complejidad de los sistemas de innovación, que obliga continuamente a reformular sus dimensiones organizacionales, estructurales e institucionales, y otra cosa bien distinta es subestimar que estos cambios en la gestión política están condicionados a los intereses económicos. Esta necesaria distinción es la que permite explicar la escasa preocupación sobre aspectos tan relevantes como (Sarewitz, 1996): el uso del conocimiento adaptado a las necesidades de la humanidad, relevante por tanto como respuesta al desafío del reparto global y a la naturaleza social de los problemas; una agenda de gobierno supeditada al interés público; así como una gestión democrática del conocimiento que decida en la mediación del mercado entre el laboratorio y la sociedad. Una primera conclusión preliminar indica por tanto que los

el 1 julio de 1999, bajo el auspicio de la UNESCO (Organización de las Naciones Unidas para la Educación, la Ciencia y la Cultura) y la ICSU (International Council for Science). Una información completa se encuentra en: http://www.unesco.org/science/wcs/index.htm

${ }^{12}$ Hay estudios que amplían los propósitos de la ciencia y de esta manera proponen valores, objetivos e indicadores más complejos y robustos (Bozeman, 2007). Es sugerente el monográfico editado por Vessuri (2012) sobre los usos del conocimiento orientados a la cohesión social y la inclusión social. Los últimos años la OCDE (2010) sugiere que la ciencia y la tecnología deben alinearse para responder a "los desafíos globales", a la vez que los estudios sobre innovación muestran interés por nuevos instrumentos políticos en respuesta a "los desafíos globales". Sobre el tema recientemente se han publicado secciones especiales en las revistas Research Policy (Foray, Mowery y Nelson, 2012) y Science and Public Policy (Boden, Johnston y Scapolo, 2012). Para una primera discusión de esta nueva orientación por "los desafíos globales", véase Kallerud et al., 2013. 
cambios en los estímulos políticos de la ciencia y la tecnología están condicionados por la racionalidad económica, operan sobre la optimización de los recursos y la organización del sistema, y prevalecen sobre cualquier discusión ligada a los objetivos a perseguir.

\section{La promesa de la seguridad en cuestión}

Junto al beneficio ilimitado, la otra promesa de la actividad científica deviene la seguridad. Es más, hemos indicado el rol que el análisis del riesgo juega en las promesas institucionales de seguridad de la tecnología y su desarrollo industrial. Podemos decir que el análisis institucional del riesgo, como indican Wynne (2002) y Jasanoff (2012), completa la retórica del progreso bajo una asunción acrítica de las dinámicas de crecimiento económico y cambio tecnológico. ${ }^{13}$

El uso político del análisis del riesgo parte de dos premisas complementarias (Lash, Szerszynski y Wynne, 1996): la capacidad institucional para garantizar el desarrollo seguro de la tecnología es dado por hecho; una comprensión de los conflictos generados por la tecnología pasa por problematizar al público y los motivos que originan su aversión al riesgo. Una estrategia de análisis diferente sugiere por el contrario que las percepciones sociales son resultado del juicio que merecen los comportamientos que las instituciones tienen y los discursos que junto a los expertos adoptan (Irwin y Wynne, 1996).

En este sentido, los estudios sociales del riesgo anticipan resultados concluyentes que son útiles para nuestros propósitos (Leach, Scoones y Wynne, 2005). Así, sugerimos desplazar el eje de comprensión del riesgo real y su representación cognitiva a la valoración que merecen los expertos, sus competencias técnicas y cognitivas para proteger a la gente de los efectos adversos, la convergencia de intereses que se percibe entre expertos e instituciones y la distorsión deliberada de la información disponible, la equidad en la distribución de los riesgos, así como la respuesta percibida sobre las demandas sociales en la decisión adoptada. Estas variables sugieren no presuponer la falta de confianza como factor explicativo, tampoco asociar la desconfianza a una actitud necesariamente irracional o, a una representación cognitiva errónea del riesgo, y por el contrario preguntarnos por las dinámicas que implican la configuración y constitución de los riesgos (Lofstedt, 2008). ${ }^{14}$

Los problemas ligados a la falta de confianza, vinculados a las demandas de una mayor responsabilidad social y ambiental, señalan que las expectativas hacia las

${ }^{13}$ Esta crítica guarda relación con Beck (1995) y su tesis sobre la irresponsabilidad organizada que caracteriza la sociedad del riesgo.

${ }^{14}$ Los procesos de creación pública de la confianza y su relevancia en la gestión del riesgo tecnológico también se preguntan por los factores que inhiben la movilización social. Diversos estudios prueban la relevancia que tienen la promesa económica, la dependencia y la asimetría en las relaciones de poder. Véanse: Espluga et al., 2014; Sugiman, 2012. También de estos estudios se desprende que las actitudes y los niveles de confianza no necesariamente dependen del nivel de conocimiento sobre el riesgo. 
instituciones y sus expertos para controlar los riesgos y garantizar la seguridad no son satisfechas. Un primer elemento que constituye las expectativas como ausencia de confianza es el epistemológico. El análisis institucional del riesgo vimos que presupone un marco normativo y por extensión relaciones socio-cognitivas incuestionable (Bouder, Slavin y Lofstedt, 2007). El análisis del riesgo presupone que el conocimiento disponible anticipa los impactos negativos, y en consecuencia la certidumbre puede ser delegada automáticamente para adoptar una decisión política racional. Como han mostrado Millstone et al. (2004), la autoridad competencial de la ciencia sin embargo en ocasiones se enfrenta a sistemas y actividades que obligan la toma de decisión bajo incertidumbre (conocemos las posibles consecuencias de las distintas opciones pero el conocimiento de las probabilidades es incompleto), ignorancia (conocemos las posibles consecuencias de las distintas opciones pero nos limitamos a decir que las probabilidades son no-cero) y desconocimiento (no se conocen las posibles consecuencias, hay consecuencias sobre las que desconocemos si su probabilidad es cero o no-cero, y por tanto no hay una lista completa). En estos contextos, la interpretación alternativa sugiere que la desconfianza en parte es causada por la falta de reflexividad y sensibilidad que muestran expertos y autoridades en la regulación política de la ciencia y del cambio tecnológico.

Ciertamente, la medición del riesgo presupone un conocimiento suficiente de las probabilidades y magnitudes de aquella actividad definida como negativa. Los contextos de decisión bajo riesgo e incertidumbre difieren en términos cognitivos, políticos y éticos (Lentchs y Weingart, 2011). Esta crítica incorpora otras insuficiencias del análisis estándar (Renn, 2008). Por una parte, los estilos de razonamiento y los criterios de satisfacción deciden el proceso de identificación, estimación y valoración del riesgo, en tanto que determinan la elección de impactos que se analizan, los tipos de evidencia que se integran, la forma de interpretar los resultados disponibles, la definición de los diferentes tipos de evidencia necesarios o suficientes para sostener los distintos tipos de juicio, la elección del nivel de protección y tolerancia para establecer los grados de aceptabilidad social del riesgo y la incertidumbre. La reducción del conocimiento científico a formulaciones y estimaciones estadísticas enmascara el rol de los valores epistemológicos y metodológicos en la objetividad del conocimiento (Bonneuil y Levidow, 2012; Clark, 2013; Todt y Luján, 2014). En este sentido, un modelo de gobernanza instrumental, dirigido a remediar el percibido incremento de la desconfianza, impide una justificación sustantiva de la participación y el pluralismo, a la vez que posterga enfoques más rigurosos orientados a probar y validar aquellos valores e intereses que informan la ciencia (Eizagirre, 2011; Wilsdom, Wynne y Stilgoe, 2005). Por otra parte, el carácter técnico y aplicativo del riesgo difumina en los resultados la severidad del daño, la magnitud de las consecuencias y los efectos de segundo orden en el entorno natural y social (Renn, 2014).

Estas insuficiencias indican conclusiones preliminares de relevancia. Se debe apuntar que las cuestiones normativas también se encuentran en el ámbito de la regulación y los modos de enmarcar el riesgo y la seguridad. Por otra parte, los valores epistemológicos difícilmente pueden justificarse como la dimensión exclusiva de las cuestiones metodológicas. A su vez, ahora los objetivos y criterios prácticos se 
convierten en objeto de controversia en el interior de la comunidad de científicos y la experticia. ${ }^{15}$ La falta de seguridad y el alto nivel de incertidumbre a nivel técnico, epistemológico y metodológico, así como el carácter elevado y relevante de la apuesta en la medida que aborda cuestiones relativas al ambiente y la sociedad implican -como resuelven Levidow y Carr (2010) en su análisis de la biotecnología agroalimentariaconflictos axiológicos y choques entre diferentes modelos normativos sobre el lugar de la ciencia en la sociedad y sobre formas de vivir la vida y relacionarse con la naturaleza.

En definitiva, los problemas ligados a la confianza pueden originarse por sentimientos de dependencia que una ciencia orientada por valores y un contexto de asimetría de poder genera en la ciudadanía (Irwin, Jensen y Jones, 2013). ${ }^{16}$ La escasa confianza en la dimensión técnica además se agrava en su dimensión afectiva (Engdahl y Lidskog, 2014; Harmon, Laurie y Haddow, 2013), de tal manera que el discurso de la controlabilidad y la predecibilidad se perciben como irreflexivos y así la cultura científica de asesores institucionales entra en conflicto con las identidades ciudadanas (Glerup y Horst, 2014; Jasanoff, 1990; Maasen y Weingart, 2005). Se trataría de dinámicas de dependencia en las que confluyen dimensiones epistemológicas, políticas y socioculturales, que evidencian un desajuste con relación a las promesas de seguridad y control (Rosa, Renn y McCright, 2014).

\section{Consideraciones finales}

Las PCTI establecen una asociación secuencial y triunfalista entre investigación básica, aplicación tecnológica, beneficio ilimitado y escenarios seguros, lo que también compromete la gestión política exclusivamente al ámbito del mercado y el eslabón de la comercialización. De esta manera, las distintas formas de gobierno de la ciencia han ajustado sus modificaciones sobre los productos generados y no sobre el proceso científico-tecnológico y los intereses que concurren en su instrumentalización y regulación. En segundo lugar, los distintos modelos reducen el problema a la evaluación de los efectos adversos cognoscibles, de tal manera que los marcos analíticos se desatienden de una concepción de la gobernanza comprometida con el ajuste de los

${ }^{15}$ La teoría de la sociedad del riesgo mundial (Beck, 2008) considera la emergencia de nuevos riesgos, caracterizados por anticipar catástrofes globales, que escapan a los métodos habituales de cálculo científico y socavan los fundamentos de las sociedades modernas. Se trata de riesgos que presentan características de lo que no puede ser indemnizado, y convierte a sus principales actores (Estado, ciencia, industria), tradicionalmente considerados como tutores, garantes y responsables de la seguridad y la racionalidad más bien en sospechosos y cómplices.

${ }^{16}$ Estas críticas pueden enlazarse con otra tradición, en línea con Escobar (2012), y así plantear como hipótesis general que la gobernanza de la ciencia en su dimensión teórica y práctica está secuestrada por presupuestos epistémicos y ontológicos característicos de la modernidad europea que dificultan captar los problemas sociales y ecológicos que nos desafían el futuro. En esta línea, nos parecen sugerentes las ideas de Wynne (Waterton, Ellis y Wynne, 2013; Wynne, 2007). 
objetivos así como de un enmarque integral de los medios seleccionados, los marcos reguladores y la verificación de los resultados.

En este artículo hemos elaborado un análisis crítico de las PCTI. De hecho, algunos de los problemas que el segundo bloque del artículo ha reconstruido se reconocen en la progresiva radicalización de las políticas de innovación responsable en la UE. Así, en el ámbito institucional en el seno de la estrategia Horizonte 2020 adquiere relevancia el lenguaje político asociado a la Investigación y la Innovación Responsables (IIRs) y se reclama que las actividades de investigación e ingeniería deben alinear mejor su proceso y resultados con los valores, las necesidades y las expectativas de la sociedad europea. ${ }^{17}$ La Unión Europea ha reconocido la importancia de considerar la dirección y el contenido de la investigación y la innovación. También otras instituciones, como revelan los recientes documentos sobre innovación de la OCDE y la estrategia de Obama, se inclinan paulatinamente a orientar la investigación y la innovación para dar respuesta a los desafíos que afectan a nuestras sociedades globales.

Este nuevo discurso sobre las PCTI se apropia de un enfoque basado en los desafíos sociales y globales (el cambio climático, el envejecimiento de la población, la escasez de recursos) y postula una reorganización de la actividad científico-tecnológica como respuesta a las cuestiones planteadas. En este sentido, el enfoque IIRs amplía su propuesta y propone que todos los actores societales (investigadores, ciudadanos, decisores políticos, empresas, organizaciones del tercer sector, etc.) ... [deben] conjuntantemente participar durante todo el proceso de investigación e innovación para alinear mejor tanto el proceso como los resultados, con los valores, las necesidades y las expectativas de la sociedad europea' ${ }^{18}$ Esta concepción de la innovación no se justifica sobre premisas acríticas y asunciones macroeconómicas, y en contraposición a modelos que reducían la evaluación a beneficios de mercado y riesgos anticipados, por el contrario sugiere ampliar los indicadores de evaluación a los impactos justos y deseados (von Schomberg, 2013: 51).

La emergencia de nuevos conceptos (gobernanza, innovación responsable, desafíos globales) y su integración en la narrativa de las PCTI europeas y nacionales abre una serie de interrogantes. Algunas de ellas están íntimamente ligadas al propio enfoque IIRs. Debemos plantear las siguientes cuestiones: ¿cómo diseñar políticas que aspiren a institucionalizar la valorización de la investigación y la innovación como motor de la competitividad económica, junto con la incorporación de las dimensiones ambientales, éticas y sociales en la constitución del conocimiento validado? ¿cómo formalizar procesos de investigación e innovación responsables, instigados desde su inicio en la definición y luego en la progresión, dirección, deliberación y legitimación, por la multidisciplina y atenta también a las ciencias sociales y humanas? ¿cuáles son los

${ }^{17}$ EC. 2012. Responsible Research and Innovation: Europe's ability to respond to societal challenges. https://ec.europa.eu/research/swafs/pdf/pub_rri/KI0214595ENC.pdf [Acceso: 1712-2013].

${ }^{18}$ EC. 2013. Horizon 2020, Work Programme 2014-2015: 16. Science with and for Society, C (2013) 8631, Brussels, p.4. 
impactos adecuados y justos de la actividad científico-tecnológica y sobre qué valores deben anclarse?

En todo caso, el impulso prescriptivo de la innovación, concebida primariamente como elemento para la competitividad económica, se muestra resistente al cambio, como puede apreciarse en buena parte de los documentos sobre la Unión de la Innovación, ${ }^{19} \mathrm{e}$ indica una tensión de base que los nuevos enfoques sobre investigación e innovación responsables, que demandan una mayor apertura en los procesos de innovación, van a tener que afrontar. En la misma línea, en el documento (The Lund Declaration, 2009) en el que se asentaron las bases conceptuales de la perspectiva de los "grandes desafíos" adoptada en la política científica de la UE se observa que la nueva estrategia puede ahondar en la progresiva tecnocientificación de la vida y el mundo, a la vez que re-introduce la innovación como atemperador de los conflictos sociales y culturales. ${ }^{20}$ Por otra parte, a esta creciente institucionalización de la responsabilidad precede la controversia de la biotecnología agroalimentaria. Aquel fracaso demostró que la innovación tecno-industrial debe necesariamente mostrarse sensible a las preocupaciones sociales ligadas a los conocimientos, intereses, objetivos y regulaciones que intervienen en el cambio socio-técnico. De alguna manera, la integración de enfoques IIRs puede interpretarse como una respuesta institucional a la experiencia de que innovaciones económicamente viables pueden fracasar al ser cuestionadas socialmente. Esto abre la interrogante sobre la legitimación y el carácter sustantivo o superficial e instrumental de la transformación que promueve el enfoque IIRs.

El análisis de las PCTI europeas no puede descuidar las dinámicas científicotécnicas, políticas, económicas y sociales por las cuales se aplican (y se dejan de aplicar) enfoques de tipo IIR en los diseños de las agendas de I+D. En otras palabras, también en las PCTI el significado, alcance y aplicación de las diferentes alternativas depende de las dinámicas económicas, políticas y sociales por las cuales se instrumentalizan y regulan la ciencia y la tecnología. En otras palabras, el modo como representamos y enmarcamos la gobernanza de la investigación y la innovación decidirá muchos problemas epistemológicos y normativos, la calidad del espacio público así como nuestras acciones posibles y deseables. En este artículo hemos identificado buena parte de los aspectos que pueden explicar las posibilidades y los límites de la nueva retórica asociada a "la investigación como respuesta a los desafíos globales". Se trata de un asunto que se alimenta simultáneamente por nuevos horizontes, narrativas sociotécnicas alternativas y viejos problemas irresueltos.

${ }^{19}$ COM. 2010. A Rationale for Action: Europe 2020 Flagship Initiative. Innovation Union. 6.10.2010. SEC(2010) 1161 final, Brussels.

${ }^{20}$ En la declaración puede leerse: "Meeting the Grand Challenges will be a prerequisite for continued economic growth and for improved chances to tackle key issues. It will involve women and men on equal terms in the development of society and cut across social, religious, generational and cultural obstacles bringing about new possibilities and increase the well being and quality of life for all". (Lund Declaration 2009). http://www.vinnova.se/upload/dokument/ Verksamhet/UDI/Lund_Declaration.pdf [Acceso: 17-12-2013] 


\section{Bibliografía}

Barben, D. (2007): "Changing Regimes of Science and Politics: Comparative and Transnational Perspectives for a World in Transition", Science and Public Policy, 34 (1), pp. 55-69.

Bauer, M. (2015): Atoms, Bytes \& Genes: Public Resistance and Techno-Scientific Response, London, Routledge.

Bauer, M., R. Shukla y N. Allum (eds.) (2012): The Culture of Science: How the Public Relates to Science Across the Globe, London, Routledge.

Beck, U. (1995): Ecological Politics in an Age of Risk, Cambridge, Polity Press.

Beck, U. (2008): La sociedad del riesgo mundial: en busca de la seguridad perdida, Barcelona, Ediciones Paidós Ibérica.

Bernal, J.D. (2010): The Social Function of Science, London, Faber \& Faber.

Berman, E.P. (2012): Creating the Market University: How Academic Science Became an Economic Engine, Princeton, Princeton University Press.

Berman, E.P. (2014): "Not Just Neoliberalism: Economization in U.S. Science \& Technology Policy", Science, Technology, \& Human Values, 39, pp. 397-431.

Besley, J. (2013): "The state of public opinion research on attitudes and understanding of science and technology", Bulletin of Science, Technology, and Society, 33 (1/2), pp. 12-20.

Boden, M., R. Johnston y F. Scapolo (eds.) (2012): "The Role of FTA in Responding to Grand Challenges", Science and Public Policy, special section, 39 (2), pp. 135-283.

Bonneuil, C. y L. Levidow (2012): "How does the World Trade Organization know? The mobilization and staging of scientific expertise in the GMO trade dispute", Social Studies of Science, 42 (1), pp. 75-100.

Bouder, F., D. Slavin y R. Lofstedt (2007): The Tolerability of Risk. A New Framework for Risk Managament, London, Routledge.

Bozeman, B. (2007): Public Values and Public Interest, Washington, DC, Georgetown University Press.

Bozeman, B. y D. Sarewitz (2011): "Public Value Mapping and Science Policy Evaluation", Minerva, 49, pp. 1-23.

Brown, N. (2003): "Hope Against Hype: Accountability in Biopasts, Presents and Futures", Science Studies, 16 (2), pp. 3-21.

Brown, N. y M. Michael (2003): "A Sociology of Expectations: Retrospecting Prospects and Prospecting Retrospects", Technology Analysis and Strategic Management, 15 (1), pp. 3-18.

Brown, N., M. Borup, K. Konrad y H. van Lente (2006): “The Sociology of Expectations in Science and Technology", Technology Analysis and Strategic Management, 18 (3/4), pp. 285-444.

Bush, V. (1945): Science: The Endless Frontier, Washington, D.C., NSF.

Clark, L.F. (2013): "Framing the uncertainty of risk: Models of governance for genetically modified foods", Science and Public Policy, 40 (4), pp. 479-491.

Coenen, C. y E. Simakova (eds.) (2013): "Governance of Technovisionary Sciences", Science, Technology \& Innovation Studies, special section, 9 (2), pp. 3-103. 
Coozens, S., E. Kallerud, L. Ackers, B. Gill, J. Harper, T. Santos Pereira y N. ZarbAdami (2007): Problems of Inequality in Science, Technology, and Innovation Policy, Project Deliverable \#2, James Martin Institute for Science and Civilization.

Dickson, D. (1984): The New Politics of Science, New York, Pantheon.

Douglas, M. y A. Wildavsky (1982): Risk and Culture: An Essay on the Selection of Technological and Environmental Dangers, Berkeley, University of California Press

Eizagirre, A. (2011): "La precaución como principio de acción sostenible", Isegoría, 44, pp. 303-324.

Eizagirre, A. (2013): "Las percepciones sociales en Europa sobre el rol de la ciencia y la tecnología", Revista de Estudios Sociales, 47, pp. 67-78.

Elzinga, A. (2012): "Features of the current science policy regime: Viewed in historical perspective", Science and Public Policy, 39 (4), pp. 416-428.

Elzinga, A. y A. Jamison. 1995. "Changing Policy Agendas in Science and Technology", en S. Jasanoff, G. E. Markle, J.C. Peterson y T. Pinch (eds.), Handbook of Science and Technology Studies, 572-597. London: Sage.

Engdahl. E. y R. Lidskog (2014): "Risk, communication and trust: Towards an emotional understanding of trust", Public Understanding of Science, 23 (6), pp. 703-717.

Escobar, A. (2012): "Más allá del desarrollo: postdesarrollo y transiciones hacia el pluriverso", Revista de antropología social, 21, pp. 23-62

Espluga Trenc, J., J. et al. (2014): "Factores que inhiben la movilización social: el caso del área petroquímica de Tarragona", Revista Española de Investigaciones Sociológicas, 146, pp. 191-216.

Felt, U. (2007): Science and governance: Taking European knowledge society seriously, Brussels: European Commission, EUR 22700.

Foray, D., D. Mowery y R. Nelson (eds.) (2012): "The need for a new generation of policy instruments to respond to the Grand Challenges", Research Policy, special section, 41 (10), pp. 1697-1792.

Glerup, C. y M. Horst (2014): "Mapping Social Responsibility in Science”, Journal of Responsible Innovation, 1 (1), pp. 31-50.

Godin, B. (2005): Science, Accounting and Statistics: the Input-Output Framework. Quebec, Project on the History and Sociology of S\&T StatisticsWorking Paper No. 31.

Godin, B. (2007): "Science, Accounting and Statistics: The Input-Output Framework", Research Policy, 36 (9), pp. 1388-1403.

Godin, B. (2010): The Making of Science, Technology and Innovation Policy: Conceptual Frameworks as Narratives - 1945-2005, Montreal, Centre Urbanisation Culture Société.

Godin, B. (2015): Innovation Contested: The Idea of Innovation over the Centuries, London, Routledge.

Godin, B. \& C. Doré (2005): Measuring the Impacts of Science: Beyond the Economic Dimension, Montreal, Centre Urbanisation Culture Société.

Godin, B. y J.P. Lane (2013): "Pushes and Pulls': The Hi(story) of the Demand Pull Model of Innovation", Science, Technology and Human Values, 38(5), pp. 621-54. 
Golding, D. (1992): “A Social and Programmatic History of Risk Research”, en S. Krimsky y D. Golding (eds.), Social Theories of Risk, pp. 23-52, Westport, CT: Greenwood Publishing Group.

Hackett, E.J. (1990): "Science as a Vocation in the 1990s: The Organizational Culture of Academic Science", Journal of Higher Education, 61(3), pp. 241-279.

Harmon, S., G. Laurie y G. Haddow (2013): "Governing risk, engaging publics and engendering trust: New horizons for law and social science?", Science and Public Policy, 40 (1), pp. 25-33.

Horst, M. (2007): "Public Expectations of Gene Therapy: Scientific Futures and their Performative Effects on Scientific Citizenship", Science, Technology and Human Values, 32 (2), pp. 150-171.

Irwin, A. y B. Wynne (1996): Misunderstanding Science? The Public Reconstruction of Science and Technology. Cambridge, Cambridge University Press.

Irwin, A., T.E. Jensen y K. Jones (2013): "The good, the bad and the perfect: criticizing engagement practice", Social Studies of Science, 4 (1), pp. 119-136.

Jamison, A. (2012): "Science and Technology in Postwar Europe", en D. Stone (ed.). The Oxford Handbook of Postwar European History, pp. 630-648, Oxford, Oxford University Press.

Jasanoff, S. (1990): The Fifth Branch: Scientific Advisors as Policymakers, Cambridge, Harvard University Press.

Jasanoff, S. (2012): Science and Public Reason, London, Routledge.

Kallerud, E. (2012): Goals conflict and goal alignment in science, technology and innovation policy discourse, NIFU Nordic Institute for Studies inInnovation, Research and Education. Disponible en: http://www.csiic.ca/PDF/Kallerud.pdf [Consulta: 17 de diciembre de 2013]

Kallerud, E. y I. Ramberg (2002): "The order of discourse in surveys of public understanding of science", Public Understanding of Science, 11 (3), pp. 213-224.

Kallerud, E., E. et al. (2013): Dimensions of research and innovation policies to address grand and global challenges, Working Paper 13/2013, Oslo, Nordisk institutt for studier av innovasjon, forskning og utdanning.

Kriebel, D. et al. (2001): "The Precautionary Principle in Environmental Science", Environmental Health Perspectives, 109 (9), pp. 871-876.

Lash, S., B. Szerszynski y B. Wynne (1996): Risk, Environment and Modernity: Towards a New Ecology, London, Sage.

Laspra, B. y E. Muñoz (eds.) (2014): Culturas cientificas e innovadoras, Progreso social, Ciudad de Buenos Aires, Eudeba.

Leach, M., I. Scoones y B. Wynne (eds.) (2005): Science and Citizens: Globalisation $\&$ The Challenge of Engagement, London, Zed Books.

Lentchs, J. y P. Weingart (eds.) (2011): The Politics of Scientific Advice, Institutional Design for Quality Assurance, Cambridge, Cambridge University Press.

Levidow, L. y S. Carr (2010): GM Food on Trial: Testing European Democracy, London, Routledge.

Lofstedt, R.E. (2008): Risk Management in Post-Trust Societies, London, Routledge. 
Maasen, S. y P. Weingart (eds.) (2005): Democratization of Expertise?: Exploring Novel Forms of Scientific Advice in Political Decision-Making, Berlin, Springer.

Marklund, G., N. Vonortas y Ch. Wessner (eds.) (2009): The Innovation Imperative: National Innovation Strategies in the Global Economy, Cheltenham. UK, Edward Elgar

McDaniels, T. y M. Small (eds.) (2004): Risk Analysis and Society: An Interdisciplinary Characterization of the Field, Cambridge, Cambridge University Press.

Miettinen, R. (2013): Innovation, Human Capabilities, and Democracy. Towards an Enabling Welfare State, Oxford, Oxford University Press.

Millstone, E. et. al. (2004): Science in trade disputes related to potential risk: comparative case studies, Sevilla, European Commission.

Moore, K., D. Kleinman, D. Hess y S. Frickel (2011): "Science and neoliberal globalization: A political sociological approach", Theory and Society, 40, pp. 505532.

Otway, H. y B. Wynne (1989): "Risk Communication: Paradigm and Paradox", Risk Analysis, 9 (2), pp. 141-145.

Polanyi, M. (1962): "The Republic of Science: Its Political and Economic Theory", Minerva 1, pp. 54-73.

Renn, O. (1992): "Concepts of risk: A clarification", en S. Krimsky y D. Golding (eds.): Social Theories of Risk, pp. 53-79, Westport, CT, Greenwood Publishing Group.

Renn, Ortwin (2008): Risk Governance. Coping with Uncertainty in a Complex World, London, Earthscan.

Renn, O. (2014): "Towards a Socio-Ecological Foundation for Environmental Risk Research", en S. Lockie, D. Sonnenfeld y D. Fisher (eds.): Routledge International Handbook of Social and Environmental Change, pp. 207-220, London, Rotledge: 207-220

Reynolds, L. y B. Szerszynski (2012): "Neoliberalism and Technology: Perpetual Innovation or Perpetual Crisis?", en L. Pellizzoni y M. Ylonen (eds.). Neoliberalism and Technoscience: Critical Assessments, pp. 27-46, Farnham, Ashgate Publishing.

Rosa, E., O. Renn y A. McCright (2014): The Risk Society Revisited. Social Theory and Governance, Philadelphia,Temple University Press.

Salomon, J-J. (2000): "L'OECD et les politiques scientifiques", Revueu pour l'histoire $d u$ CNRS, 3, pp. 40-58.

Sarewitz, D. (1996): Frontiers of Illusion: Science, Technology and the Politics of Progress, Philadelphia, Temple of University Press.

Sarewitz, D. (2007): “Does Science Policy Matter", Issues in Science and Technology, 3, pp. 31-38.

Sarewitz, D. et al. (2004): "Science Policy in Its Social Context", Philosophy Today, pp. 67-83.

Scoones, I., M. Leach y P. Newell (eds.) (2015): The Politics of Green Transformation, London, Routledge.

Selin, C. (2007): "Expectations and the Emergence of Nanotechnology", Science, Technology and Human Values, 32 (2), pp. 196-220. 
Shrader-Frechette, K. (1991): Risk and Rationality: Philosophical Foundations for Populist Reforms, Berkeley, University of California Press.

Shrader-Frechette, K. (2007): Taking Action, Saving Lives: Our Duties to Protect Environmental and Public Health, Oxford, Oxford University Press.

Slaughter, S. y G. Rhoades (2004): Academic Capitalism and the New Economy: Markets, State, and Higher Education, Baltimore, The Johns Hopkins University Press.

Stephan, P. (2012): How Economics Shapes Science, Cambridge, MA, Harvard University Press.

Sugiman, T. (2012): "Lessons learned from the 2011 debacle of the Fukushima nuclear power plant", Public Understanding of Science, 23 (3), pp. 254-267.

Todt, O. (2011): "The limits of policy: Public acceptance and the reform of science and technology governance", Technological Forecasting and Social Change, 78 (6), pp. 902-909.

Todt, O. y J.L. Luján (2014): "Values and Decisions: Cognitive and Noncognitive Values in Knowledge Generation and Decision Making", Science, Technology \& Human Values September, 39 (5), pp. 720-743

Vessuri, H. (ed.) (2012): "The use of knowledge for social cohesion and social inclusion", Science and Public Policy, special section, 39 (5), pp. 545-617.

Von Roten, F. e Y. de Roten (2013): "Statistics in science and in society: From a stateof-the-art to a new research agenda", Public Understanding of Science, 22 (7), pp. 768-784.

Von Schomberg, R. (2013): “A Vision of Responsible Research and Innovation”, en R. Owen, J. Bessant y M. Heintz (eds.): Responsible Innovation: Managing the Responsible Emergence of Science and Innovation in Society, pp. 51-74, Chichester, UK, Wiley.

Waterton, C., R. Ellis y B. Wynne (2013): Barcoding Nature: Shifting Cultures of Taxonomy in an Age of Biodiversity Loss, London, Routledge.

Woodhouse, E. y D. Sarewitz (2007): "Science Policies for Reducing Societal Inequities", Science and Public Policy, 34 (2), pp. 139-150.

Wilsdon, J., B. Wynne y J. Stilgoe (2005): The Public Value of Science: Or How to Ensure that Science Really Matters, London, Demos.

Wynne, B. (2002): "Risk and Environment as Legitimatory Discourses of Technology: reflexivity inside-out", Current Sociology, 50 (3), pp. 459-477.

Wynne, B. (2007): "Indigenous Knowledge and Modern Science as Ways of Knowing and Living Nature: The Contexts and Limits of Biosafety Risk Assessment", en T. Traavik y L. Li Ching (eds.): Biosafety First. Holistic Approaches to Risk and Uncertainty in Genetic Engineering and Genetically Modified Organisms, Malaysia, Third World Network and GenØk.

Wynne, B. (2008): "Public participation in science and technology: Performing and Obscuring a Political-Conceptual Category Mistake", East Asian Science, Technology and Society: An International Journal, 1 (1), pp. 99-110.

Ziman, J. (1998): "Why Must Scientists Become More Ethically Sensitive Than They Used to Be?", Science, 282 (5395), pp. 1813-181. 